treatment with Mepoluzimab. Patients were tested for strongyloidiasis, filariasis and schistosomiasis depending on travel history. Patients symptomatic of helminth infection (e.g., diarrhoea) were excluded from this evaluation.

Results We tested 80 patients, 32 from severe asthma clinic, 42 from the general asthma clinic and 6 from other clinics. From these 16 (20\%) had positive parasite serology: 14 of these were for Strongyloides stercoralis and 1 each for filarial and schistosomal. All the positives had asthma and 4 were from the severe asthma service. The average IgE was 433 and the average eosinophil count was 0.7 . There was no statistical difference between the eosinophil counts, or total IgEs, between the positive and negative groups.

Conclusion There is a high prevalence of asymptomatic parasitic infection within our cohort, suggesting local patients who have an eosinophilia should be screened for helminth disease even in the presence of another cause eosinophilia. Furthermore, we recommend all patients being assessed for a biologic that would inhibit Th2 responses, such as Mepoluzimab, should be screened for latent Stronglyloides stercoralis infection given the danger of hyper-infection upon immunosuppression.

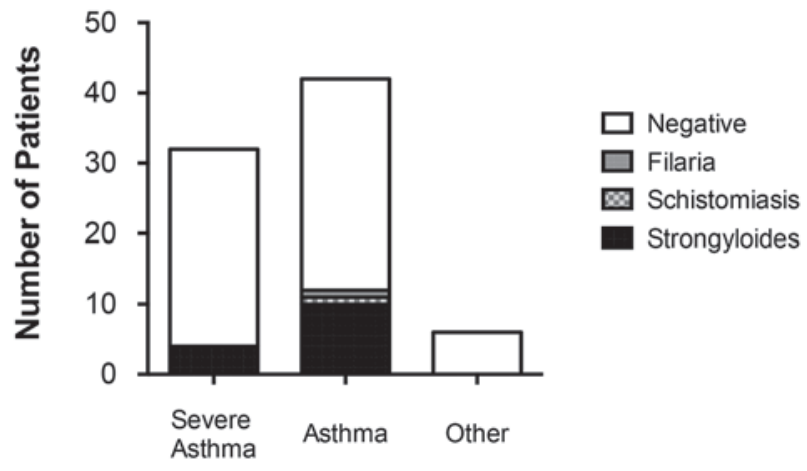

Abstract P20 Figure 1

\section{P21 EVALUATING THE CLINICAL IMPACT OF CORTICOSTEROID SENSITIVITY AND INSENSITIVITY OF PERIPHERAL BLOOD MONONUCLEAR CELLS IN SEVERE ASTHMA}

A Thomas, S Siddiqui, Y Amrani. Department of Infection, Immunity and Inflammation, University of Leicester, Leicester, UK

10.1136/thoraxjnl-2017-210983.163

Steroid insensitivity and the heterogeneous nature of asthma represent a significant clinical challenge for patients with severe asthma. The use of peripheral blood mononuclear cells (PBMC) in recent studies has allowed researchers to demonstrate that poor steroid response could be due to corticosteroid insensitivity in immune cells. These cells have been used as a model to investigate the underlying molecular mechanisms of corticosteroid resistance. These models were however inconclusive and have failed to consider asthma phenotypes. Our aim was to determine in vitro corticosteroid sensitivity of isolated PBMCs in $\mathrm{T}_{\mathrm{H}} 2$ high and $\mathrm{T}_{\mathrm{H}} 2$ low patients. Severe asthma patients as classified by the Global Initiative for Asthma were recruited and divided into $\mathrm{T}_{\mathrm{H}} 2$ high and $\mathrm{T}_{\mathrm{H}} 2$ low cohorts based on fractional exhaled nitric oxide (FeNO). Isolated PBMCs were stimulated with $\alpha \mathrm{CD} 3 / 28$ alone or in the presence of dexamethasone $\left(10^{-10} \mathrm{M}-10^{-6} \mathrm{M}\right)$. IL-5, IL-13 and IL-
17 cytokine release were measured using ELISA. Correlation studies were carried out to determine whether the $\mathrm{LogIC}_{50}$ or $\mathrm{FeNO}$ correlated with markers of asthma severity. PBMCs stimulated with $\alpha C D 3 / 28$ showed significant IL-5 and IL-17 release in $\mathrm{T}_{\mathrm{H}} 2$ low but not $\mathrm{T}_{\mathrm{H}} 2$ high patients. Production of IL-5 or IL-7 was dose-dependently suppressed by dexamethasone with different potencies. IL-13 was only significantly stimulated in $\mathrm{T}_{\mathrm{H}} 2$ low patients and did not show suppression by dexamethasone. A significant negative correlation between $\alpha \mathrm{CD} 3 / 28$ stimulated IL-17 production and FeNO was observed. Overall, PBMCs from severe asthmatics retain cell responsiveness to TCR engagement with production of $\mathrm{T}_{\mathrm{H}} 2$ and $\mathrm{T}_{\mathrm{H}} 17$ cytokines and inhibition by dexamethasone. Additional studies comparing $\mathrm{T}_{\mathrm{H}} 2$ high vs $\mathrm{T}_{\mathrm{H}} 2$ low patients are required to determine whether differences in in vitro CS response exist between these 2 categories of patients.

\section{P22 INDUCIBLE LARYNGEAL OBSTRUCTIONS CAUSING BREATHING PROBLEMS: A STUDY CLASSIFYING PATIENTS' LARYNGOSCOPIC PRESENTATIONS ACCORDING TO THE ERS/ELS/ACCP 2013 INTERNATIONAL CONSENSUS CONFERENCE NOMENCLATURE}

${ }^{1} \mathrm{C}$ Slinger, ${ }^{1} \mathrm{~J}$ Blakemore, ${ }^{2} \mathrm{R}$ Slinger, ${ }^{1} \mathrm{~A}$ Vyas. 'Lancashire Teaching Hospitals, Preston, UK;
${ }^{2}$ Lancaster University, Lancaster, UK

10.1136/thoraxjnl-2017-210983.164

Introduction and Objectives An international task force proposed the term 'Inducible Laryngeal Obstruction' (Ilo) to describe a group of conditions which, in the literature, has over 40 descriptive terms, including, vocal cord dysfunction (VCD). The resultant nomenclature describes and details a range of laryngeal manifestations that create an obstruction to inspiratory or expiratory airflow. This study aims to trial the use of the 'laryngeal findings' aspect of the nomenclature to describe features of Ilo and to gain insight into the practicality of using these definitions in clinical practice.

Method Twenty-two prospective and twenty-eight retrospective analyses of video laryngoscopic assessments for Ilo were classified in a cohort of patients referred to our Tertiary Airways service who had no uncontrolled underlying respiratory symptoms. These video-laryngeal recordings were classified according to the consensus laryngeal nomenclature. The assessments were classified according to: onset of obstruction (glottic, supraglottic, or both), phase of respiratory cycle (inspiratory, expiratory, or both), onset timing (fast or slow) and resolution of symptoms (fast or slow). These classifications were conducted by two respiratory speech and language therapists and a consultant respiratory physician with extensive experience of completing such assessments to obtain diagnosis, with consensus being achieved before final rating.

Results Forty percent of patients had combined glottic and supraglottic presentation, 31\% supraglottic and 29\% glottic only. The majority of patients (61\%) had sole inspiratory obstruction, and $67 \%$ had a fast onset of symptoms. Sixtyseven percent also had a fast resolution of symptoms; although this could not be reliably documented, as in our laryngoscopy protocol, when symptomatic, the SLT demonstrates to the patient how to reverse the obstruction with laryngeal control techniques following challenge testing to minimise patient distress, and begin therapy. 
Conclusion This classification system can be useful to accurately describe laryngoscopic findings during Ilo assessment. This system is now incorporated into our reporting practices to increase capture of Ilo diagnosis. The inclusion of supraglottic presentation with symptoms supports accurate diagnosis and treatment and further understanding of Ilo.

\section{P23 PATIENT-REPORTED ONSET FACTORS IN INDUCIBLE LARYNGEAL OBSTRUCTION}

${ }^{1} \mathrm{C}$ Slinger, ${ }^{2} \mathrm{R}$ Slinger, ${ }^{1} \mathrm{~A}$ Vyas. 'Lancashire Teaching Hospitals, Preston, UK; ${ }^{2}$ Lancaster University, Lancaster, UK

\subsection{6/thoraxjnl-2017-210983.165}

Introduction and Objectives Inducible Laryngeal Obstruction (Ilo) is poorly understood, in terms of aetiology and onset by patients and clinicians. Patients presenting to our Tertiary Airways service commonly seek an understanding of the causes and triggers of their Ilo episodes. This study aims to develop a taxonomy of categories of patient-reported onset factors for Ilo from a sample of patients with confirmed Ilo on laryngoscopy, referred to our service.

Method Within a nine month period, 103 patients referred to the Airways service $(76 \%$ female, $24 \%$ male; age range $15-86$ years $($ median $=52)$ ) with endoscopically-confirmed Ilo were asked to report historical factors contemporaneous with the initial onset of Ilo symptoms. A retrospective analysis of patient notes was also conducted to identify co-morbidities and additional onset factors. Single or multiple onset factors for each patient were collected, which were coded into initial themes. From these themes, second-order onset categories were developed which incorporated factors reported by all participants.

Results Thirteen initial onset themes were developed. These included psychological factors (25\% of patients), upper respiratory tract infections (23\%), reflux (17\%), chest infections (14\%), medical conditions (12\%) and surgery (10\%). These were then refined into a taxonomy of five categories of onset factors:

- Respiratory Tract Infections and Viruses (40\% of patients)

- Underlying Medical Conditions, e.g., rhinitis with post nasal drip, reflux (34\%)

- Psychological difficulties (25\%)

- Irritants, e.g., medication (23\%)

- Exercise (7\%)

Only one patient had onset factors in multiple categories, indicating that these categories are largely independent of each other. The prevalence of certain onset factors was mediated to some degree by age, e.g., medical conditions were more frequent factors for older participants, whereas exercise was a more common factor in younger participants.

Conclusions This study demonstrates patient-reported onset factors for Ilo can be usefully translated into a detailed taxonomy based on specific conditions/triggers. This understanding may be useful in furthering our understanding, both of common trigger and onset factors that can be communicated to patients, and may help to inform therapeutic interventions aimed at the active self-management of Ilo.

\section{Clinical update in COPD}

\section{P24 MAPPING OF END OF LIFE RECOGNITION AND PALLIATIVE CARE PROVISION IN COPD}

${ }^{1} \mathrm{HM}$ Ward, ${ }^{2} \mathrm{~A}$ Wood, ${ }^{3} \mathrm{C}$ Morrissey, ${ }^{4} \mathrm{~F}$ Hakkak. ${ }^{1}$ The Royal Wolverhampton NHS Trust, Wolverhampton, UK; ${ }^{2}$ Graphnet Health Ltd, Milton Keynes, UK; ${ }^{3}$ Wolverhampton CCG, Wolverhampton, UK; ${ }^{4}$ Compton Hospice, Wolverhampton, UK

10.1136/thoraxjnl-2017-210983.166

COPD kills about 25000 people per year in England and Wales (DoH 2011). NICE guidelines (2010) suggest patients with end stage COPD should have access to palliative services. The national COPD audit showed on-site palliative care provision has increased from 50\% in 2008 to $87 \%$ in 2014 but less is known about out of hospital provision. The Gold Standard Framework (GSF) prognostic indicators assist clinicians to identify patients who are approaching end of life at an earlier stage enabling appropriate interventions to take place. These patients are more likely to receive well-coordinated and high quality care (GSF/RCGP 2011). The aim of our study was to map the number of QoF registered COPD patients, the frequency of COPD specific GSF indicators, the number included on primary palliative care registers ( $\geq 2$ GSF prognostic indicators) and the number reviewed by palliative care.

Methods A multidisciplinary group with membership from CCG, acute trust, hospice and the community team oversaw the project. After a data sharing agreement was completed, data was collated from across all health care sites in our area for COPD patients enabling us to confirm the number and type of GSF prognostic indicators for each patient and healthcare activity including palliative care reviews.

Results As of March 2016 there were 4999 COPD patients; $52 \%$ were male with an average age of 69.2 years. $25.7 \%$ of the patients $(n=1285)$ had $\geq 1$ GSF prognostic indicator. The most common indicator was MRC dyspnoea score of $4 / 5$ (65.5\%), followed by body mass index <20 (25.7\%). Of the 294 patients with $\geq 2$ GSF prognostic indicators $14.6 \%$ were on the GP palliative care register. $19.0 \%$ of the 294 patients had been reviewed by the palliative care team.

Conclusion GSF prognostic indicators in COPD are prevalent with breathlessness being the most common. Only a small proportion of appropriate patients were included on the palliative care register (14.6\%) with more being seen by palliative care teams than on the registers. Further work is needed to ensure effective communication and education is provided across the whole health care system to identify patients earlier who are approaching end of life.

\section{P25 LIVING WITH COPD: A PUBLIC AWARENESS AND SCREENING CAMPAIGN}

${ }^{1} \mathrm{MG}$ Crooks, ${ }^{2} \mathrm{~J}$ Thompson, ${ }^{3} \mathrm{~S}$ Platten, ${ }^{4} \mathrm{C}$ Evans, ${ }^{2} \mathrm{~S}$ Faruqi. ${ }^{1}$ Hull York Medical School, Hull, UK; ${ }^{2}$ Hull and East Yorkshire Hospitals NHS Trust, Hull, UK; ${ }^{3}$ City Health Care Partnership, Hull, UK; ${ }^{4}$ British Lung Foundation, London, UK

10.1136/thoraxjnl-2017-210983.167 